\title{
Cb-TRAM: Tracking and monitoring severe convection from onset over rapid development to mature phase using multi-channel Meteosat-8 SEVIRI data
}

\author{
T. Zinner, H. Mannstein, A. Tafferner
}

With 14 Figures

Received 10 April 2006; Accepted 29 January 2008

Published online 26 May 2008 (C) Springer-Verlag 2008

\begin{abstract}
Summary
Cb-TRAM is a new fully automated tracking and nowcasting algorithm. Intense convective cells are detected, tracked and discriminated with respect to onset, rapid development, and mature phase. The detection is based on Meteosat-8 SEVIRI (Spinning Enhanced Visible and InfraRed Imager) data from the broad band high resolution visible, infra-red $6.2 \mu \mathrm{m}$ (water vapour), and the infra-red $10.8 \mu \mathrm{m}$ channels. In addition, tropopause temperature data from ECMWF operational model analyses is utilised as an adaptive detection criterion. The tracking is based on geographical overlap between current detections and first guess patterns of cells predicted from preceeding time steps. The first guess patterns as well as short range forecast extrapolations are obtained with the aid of a new image matching algorithm providing complete fields of approximate differential cloud motion. Based on these motion vector fields interpolation and extrapolation of satellite data are obtained which allow to generate synthetic intermediate data fields between two known fields as well as nowcasts of motion and development of detected areas. Examples of the application of Cb-TRAM and a comparison to precipitation radar and lightning data as independent data sources demonstrate the capabilities of the new technique.
\end{abstract}

Correspondence: Tobias Zinner, Deutsches Zentrum für Luft- und Raumfahrt (DLR), Institut für Physik der Atmosphäre, Oberpfaffenhofen, 82234 Wessling, Germany (E-mail: tobias. zinner@dlr.de)

\section{Introduction}

Thunderstorms have always been one of the fundamental meteorological hazards to society and economy. Ground and air transport, the construction and energy industry as well as all kinds of outdoor activities depend on early warnings and correct forecasts of thunderstorm development and propagation.

Although routine numerical forecasts operated by the weather services show some skill in forecasting the propagation of thunderstorm complexes connected to synoptic disturbances they, in general, cannot predict the onset and path of individual thunderstorms. Main reasons are the inaccurate representation of the atmospheric state at small scales in the model fields, in particular moisture and hydrometeor distributions, as well as the dynamic and microphysical state of clouds. In addition, operational grid resolution is far from being able to simulate cloud physics processes directly. Furthermore thunderstorms are generated and propagated by an often complex interaction of processes on various scales, e.g., large scale forcing by synoptic scale systems, boundary layer processes like convection and turbulence, and energy conversion at the 
ground due to the fluxes of solar radiation, heat, and moisture.

Short range forecasting of up to about 2 to $6 \mathrm{~h}$, also referred to as "nowcasting", is therefore mainly based on an appropriate extrapolation of near real-time observations in time. Starting from observations, e.g., of lightning, precipitation patterns in radar data, or of cloud patterns in satellite data, individual thunderstorms are detected and tracked. Future position and growth is then estimated using information from past development sometimes using complementary data from numerical models or conceptual convective lifecycle models.

A large number of nowcasting tools has been developed during the past 20 years providing short range warnings of strong winds and heavy precipitation, as well as convective cloud system life-cycle climatologies. Very early there have been first attempts to determine the motion of precipitation echoes from two consecutive radar images and to obtain an extrapolation of their position for the near future (e.g., Ligda 1953). Soon after satellite imagery was utilised to derive cloud motions as well (e.g., Fujita 1969). Still most current techniques are based on these two sources of near real-time information.

A group of radar echo tracking techniques has been developed, e.g., TITAN (Dixon and Wiener 1993), COTREC/TREC (Mecklenburg et al. 2000), CONRAD (Lang 2001), TRACE3D (Handwerker 2002), TRT (Hering et al. 2004), or in combination with lightning data in (Steinacker et al. 2000). The advantage of using real-time radar data for storm tracking is given by the fact that precipitation echoes allow to detect the active convective cores within the thunderstorm reliably. However, there is no possibility to detect a developing convective cell before the precipitation stage is reached. Also, radar networks providing more than a local coverage are still limited to Western Europe and North America and even there complete spatial radar coverage is not guaranteed, mainly due to ground shielding.

The alternative source of near real-time information providing global data coverage is satellite data. The detection of convectively active areas for this type of data is a more difficult task compared to radar echoes. This is mostly accomplished on the basis of temperature thresholds in thermal infra-red observations where the coldest and highest cloud tops, presumably of convective origin, can be distinguished from warmer more shallow clouds. Examples are, e.g., Machado et al. (1998), Riosalido et al. (1998), MASCOTTE (Carvalho and Jones 2001), RDT (Morel and Senesi 2002), Bolliger et al. (2003), or Feidas and Cartarlis (2005).

The information on the thunderstorm cells' motion gained from the above mentioned tracking techniques is then widely used to forecast their future location and intensity, often in an operational way by the national weather services. In addition there is an increasing effort to combine the advantages of radar and satellite data with other near real-time observations like lightning data, as well as numerical or conceptual life-cycle models to form so called "expert systems" (e.g., UK Met Office GANDOLF Pierce et al. 2000; NCAR Auto-Nowcast Mueller et al. 2003).

In this paper, a new tool for the early detection of thunderstorm development and the tracking of convection of intense activity is introduced: The Cb-TRAM algorithm (Cumulonim Bus TRacking And Monitoring), which is based on satellite data of the Meteosat- 8 SEVIRI instrument. The presented algorithm concentrates on the exploitation of the novel capabilities offered by the Meteosat Second Generation/Meteosat-8 SEVIRI instrument, to detect convection before the onset of precipitation and in regions without radar coverage.

The above listed satellite based tools are almost exclusively based on data from a thermal infra-red channel at $11 \mu \mathrm{m}$ (IR) thereby offering a detection which is insensitive to day and night differences. The detection mostly uses a single fixed temperature threshold for the IR brightness temperature between 218 and $245 \mathrm{~K}$. Thus the techniques have problems to account for different synoptic or seasonal situations and to discriminate convective and non-convective cloud systems. Convection warmer than the temperature threshold is missed.

To improve some of these issues, this work emphasises the early detection of potentially convective activity, the detection of rapid developments in storm cells still warmer than the classical thresholds, and the separation of areas of severe convective activity from extensive cirrus shields. For this purpose, additional information from the SEVIRI high resolution visible 


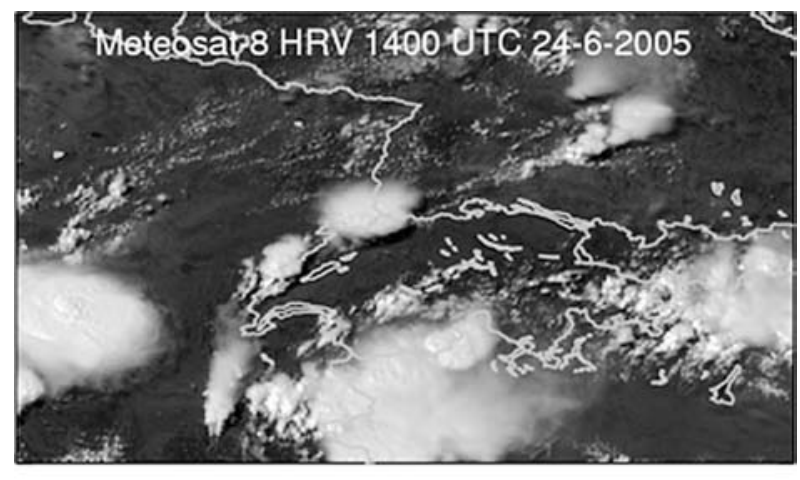

a

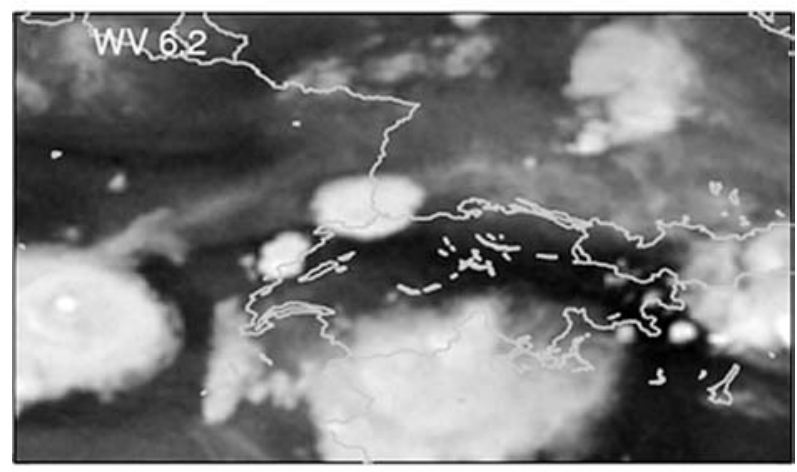

b

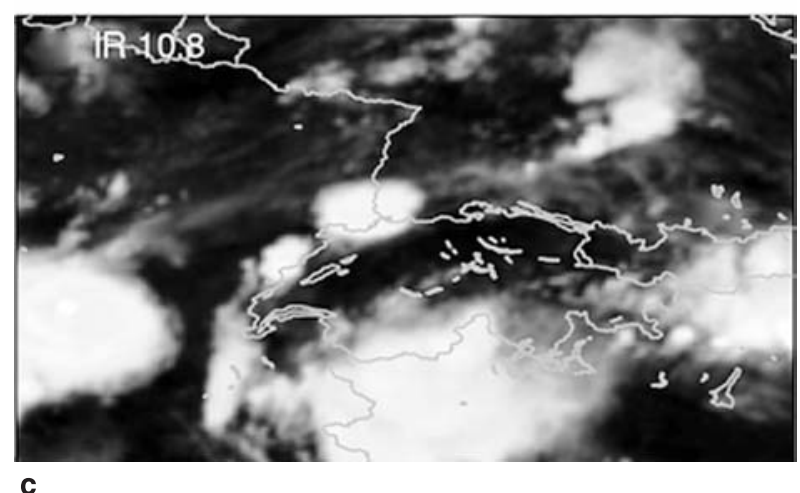

Fig. 1. Meteosat-8 SEVIRI data from 24 June 2005, (a) 14 UTC, channels: broad-band HRV (resolution about $1.5 \mathrm{~km}$ for Central Europe), (b) WV $6.2 \mu \mathrm{m}$ and (c) IR $10.8 \mu \mathrm{m}$ (both at standard resolution of about $5 \mathrm{~km}$ per pixel)

channel (HRV) as well as the infra-red $6.2 \mu \mathrm{m}$ "water-vapor" channel (WV) is used. As reflected solar radiation is used an adaption of the detection algorithm moving from daylight to nighttime is therefore unavoidable. This allows for an optimal exploitation of satellite data regarding the detection of early stages of convection, which is mostly triggered during daytime, and discrimination of convectively active areas within inactive cloud fields. In addition, the above mentioned fixed threshold principle is replaced by an adaptive technique using tropopause temperature from short range numerical weather forecasts or ECMWF operational analysis data.

The Cb-TRAM tool consists of the following parts:

(1) Central to the algorithm is the extraction of a general transformation or disparity vector field from two consecutive satellite images describing the cloud motion and local cloud developments.

(2) The detection analyses the current satellite data from three channels and identifies cell patterns of convective activity.

(3) The tracking and monitoring (TRAM) links detected cell patterns at different time steps with each other and creates a cell history $\log$ file with information on the detected patterns.

(4) The displaying routine reads this file and plots selectable information, like a cell pattern's extent, its past track, or the extrapolated position on top of the current satellite image.

Here and throughout the paper the term "cell pattern" is used for a convective object detectable by our satellite algorithm. In fact, it can contain more than one real convection cell since the technique is limited to the spatial resolution and information available from the satellite instrument.

This algorithm is presented in Sect. 2. The detection of rapid convective developments or mature thunderstorm cell patterns is described in Sect. 3. Section 4.1 presents the techniques used to track the identified cell patterns. The plotting routine is demonstrated in Sect. 5 together with some examples of application including a comparison of the detected convective cell patterns with precipitation radar and lightning data. Figure 1 shows an example of the data basis, the HRV (broad-band visible), WV (infra-red $6.2 \mu \mathrm{m}$ ), and IR (infra-red $10.8 \mu \mathrm{m}$ ) SEVIRI data. The satellite data is provided in the form of solar reflectivities or infra-red brightness temperatures.

\section{Extraction of the "motion" field}

The initial step within the detection and tracking routine is the determination of a motion vec- 


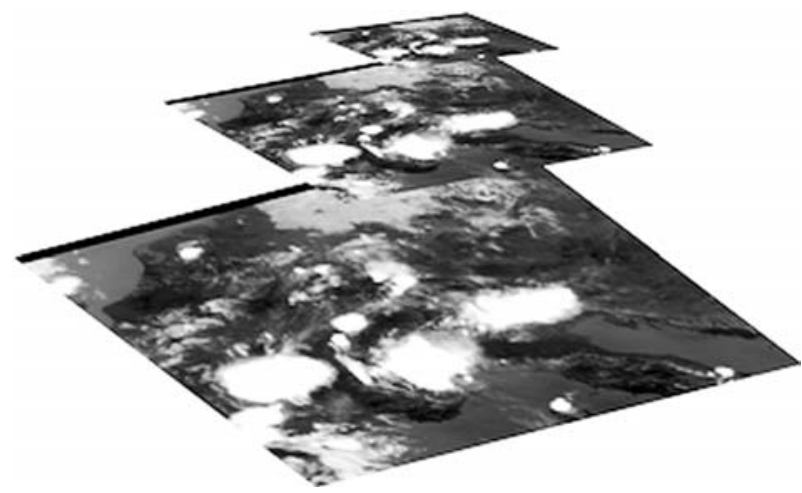

Fig. 2. Pyramid with three levels (i.e., number of subsampling levels $N=2$ ): On the topmost level (dimensions $n_{\mathrm{x}} / 4, n_{\mathrm{y}} / 4$ ) the large scale disparity vector field is obtained. It is then successively refined stepping through the other levels (dimensions $n_{\mathrm{x}} / 2, n_{\mathrm{y}} / 2$ and original dimensions $n_{\mathrm{x}}, n_{\mathrm{y}}$ )

tor field from two consecutive images. This problem is identical to the derivation of disparities from stereo pairs of images, only the interpretation differs: In our case the disparities result from cloud motion, not from changes of the view point. In analogy to the visual interpretation the first automated image matchers, like those still used in cloud motion vector determination (Schmetz et al. 1993; Menzel 2001), were feature based. Interesting cloud patterns in the first image are selected and searched for in a target area within the second one. The advantage of such matchers is that the computing effort is concentrated on a few items. The obvious disadvantage is that only a few individual motion vectors are derived and that each of these motion vectors is derived independently from the others. As computation speed is no longer a limiting factor, here an area based image matcher is used. The result of the matcher is a disparity vector field $\vec{V}$ defined at each pixel position $\vec{P}$ of the image often called "optical flow". This field $\vec{V}$ describes the warping of one of the images onto the other in a way that either the difference of the image intensities is minimised (classical optical flow) or the local correlation is maximised. A variety of different ways to calculate the optical flow can be found in literature (Lucas and Kanade 1981, Horn and Schunck 1981). In the following our implementation of a general purpose matcher is described.

\subsection{Pyramidal matcher}

Clouds and cloud fields show a strong spatial autocorrelation and often the motion of small scale features is dominated by the large scale flow. In order to take this scale dependency into account, image pairs are successively analysed from low to high resolution - a pyramidal scheme (Fig. 2). In Fig. 3a and b, an example for a simple moving structure is given. Initially the images $A$ and $B$ are extended to dimensions of a multiple of $2^{N}$, with $N$ being the number of additional sub-sampling levels of the pyramid, in both image dimensions to allow for the stepwise (pyramidal) processing. The images $A$ and $B$ are sub-sampled to the selected dimensions for all pyramid levels (compare Fig. 2).

The disparity vector $\vec{V}$ field between the two sub-sampled images is now determined by successively shifting image $A$ to $A^{s}=A(\vec{P}+$ $\left.\Delta \vec{S}_{i, j}\right)$ by $\Delta \vec{S}_{i, j}=-2 . .2$ pixels in both dimensions and comparing it to image $B$. Figure 4 shows the results for the 25 comparisons of the topmost level of our example. For each image pixel $\vec{P}$ the resolution dependent incremental shift $\Delta \vec{S}_{i}^{\text {fit }}$ and $\Delta \vec{S}_{j}^{\text {fit }}$ that provides the best fit of $A^{s}$ to image $B(\vec{P})$ is stored in $\vec{V}$.

Implemented are two different quality criteria for the best fit:

- (default) the minimum of the squared difference of the intensities (which might be either reflectivities or brightness temperatures) in a local surrounding of the image pixel of interest or

- the maximum of a localised correlation coefficient.

The localisation of both fields of quality criteria is obtained by weighting with a Gaussian filter (Fig. 5). That means, the first criterion is simply the low pass filtered squared difference calculated as the convolution

$d_{\mathrm{loc}}=\left(A^{s}-B\right)^{2} \otimes K e$

with $K e$ being the Gaussian Kernel. According to this criterion those pixels in Fig. 4 are marked, which show the lowest $d_{\mathrm{loc}}$ of all 25 comparisons.

To describe the localised correlation coefficient, it is necessary to introduce the localised 


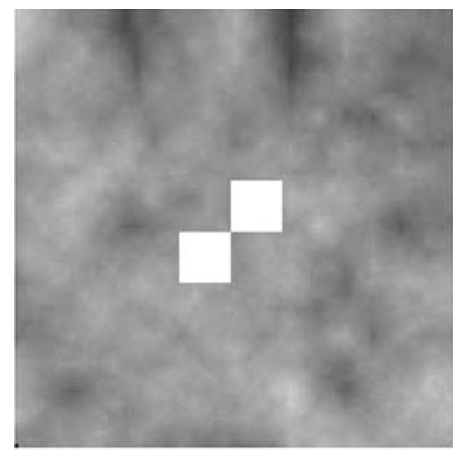

a

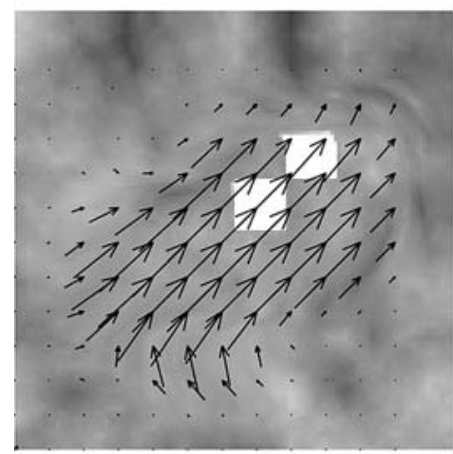

C

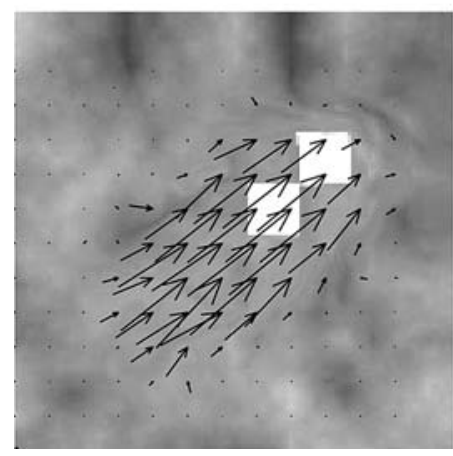

e

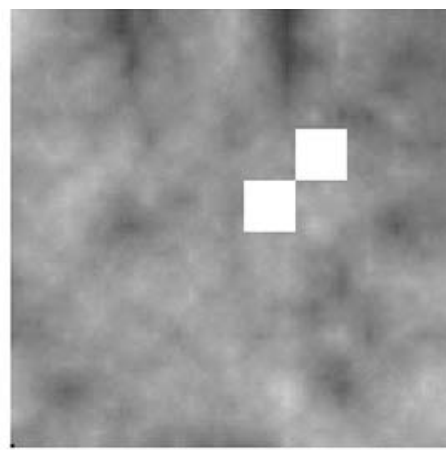

b

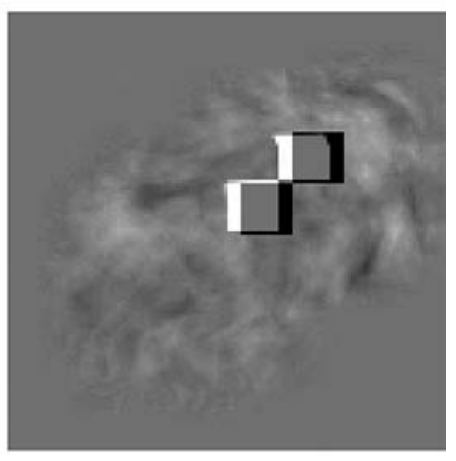

d

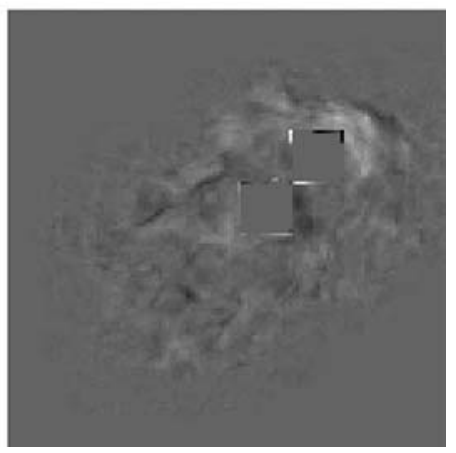

$\mathbf{f}$
Fig. 3. Pair of images $A$ and $B(\mathbf{a}, \mathbf{b})$ showing a bright structure of 2 squares on a random background. For these disparity vectors $\vec{V}$ are derived on the topmost pyramid level and plotted on top $A^{\prime}(\vec{P})=A(\vec{P}-\vec{V})$ (c). The vectors start at the location within A from where the intensity is obtained, only 1 out of 100 vectors is displayed. Using these disparities from the top most pyramid level the remaining difference field is $A^{\prime}-B(\mathbf{d})$. After processing on all pyramid levels the final disparity field (e) is obtained. The remaining difference (f) is minimal version of the mean $\bar{A}=A \otimes K e$ and the localised "standard deviation" of the image $A$ as

$S(A)=\sqrt{(A-\bar{A})^{2} \otimes K e}$.

Then the local correlation coefficient $c_{\text {loc }}$ for the images $A$ and $B$ reads as

$c_{\mathrm{loc}}=\frac{[(A-\bar{A}) *(B-\bar{B})] \otimes K e}{S(A) * S(B)}$.

The " $*$ " symbolises a point-wise multiplication operation.

These localised rotational symmetric versions of mean, standard deviation, and correlation coefficient have first been used in Mannstein et al.
(1999) for other image processing tasks. Their advantage over the use of unweighted rectangular areas for the calculation of these quantities is that artificial high frequency artefacts (from the rectangular filter edges) are suppressed. In addition the performance of the matcher does not vary between the along-axis and diagonal image directions.

Using one of the localised criteria, $d_{\mathrm{loc}}$ for all following applications, the disparity vector field $\vec{V}$ is obtained, initially for the topmost pyramid level (Fig. 3c). If no extremum is found, a zero displacement is assumed. Applying the vector field, smoothed, scaled and interpolated to the 

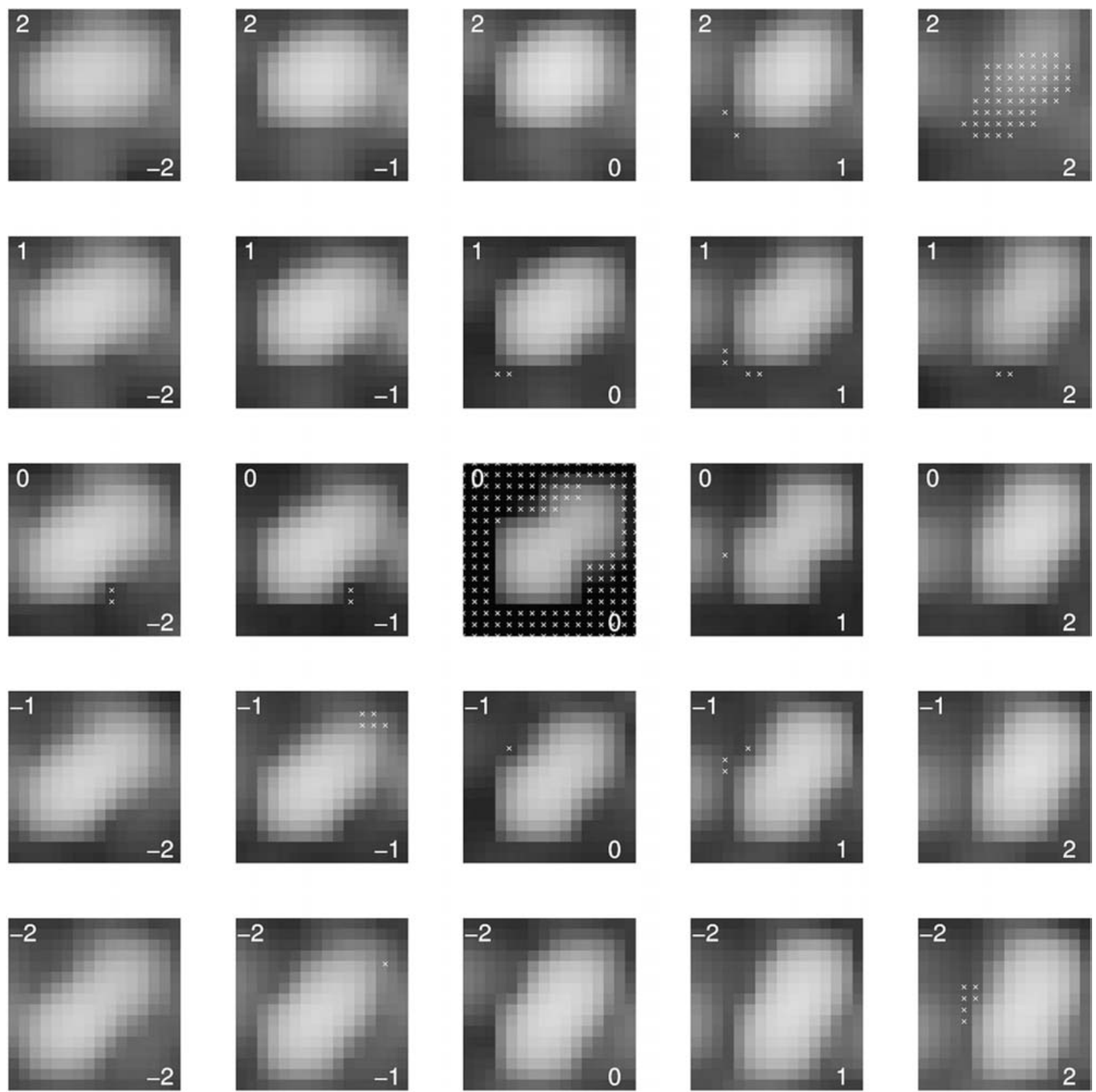

Fig. 4. The localised quality of fit between image $A$ (see Fig. 3a) shifted by -2 to 2 pixels in both dimensions and image $B$ in the topmost pyramid level. Black refers to a perfect fit. Marked are those pixels, which give the best fit. The disparity vector $\vec{V}$ field is obtained from their shift values which are indicated in upper left and lower right corners, e.g. the top right image is the quality field of a shift by 2 pixels in both positive x- and y-directions of image $A$ relative to image $B$

original image resolution, the image $A$ is warped by creating

$A^{\prime}(\vec{P})=A(\vec{P}-\vec{V})$.

Figure $3 \mathrm{c}$ shows the warped image $A^{\prime}$ and the disparity vector $\vec{V}$ after the topmost pyramid processing. The test structure of two adjacent squares is shifted to the approximate target position $3 \mathrm{~d}$. As the matching starts at a low resolu- tion, the whole cloud structure is shifted. Matching without sub-sampling the image resolution (without pyramid levels) would limit the maximum area of detectable displacement, called the "search radius" in traditional matchers, and thereby lead to erroneous disparity vectors: the top right square object in Fig. 3a would be matched to the lower left square in Fig. 3b. The interpolation also creates non-integer values for 


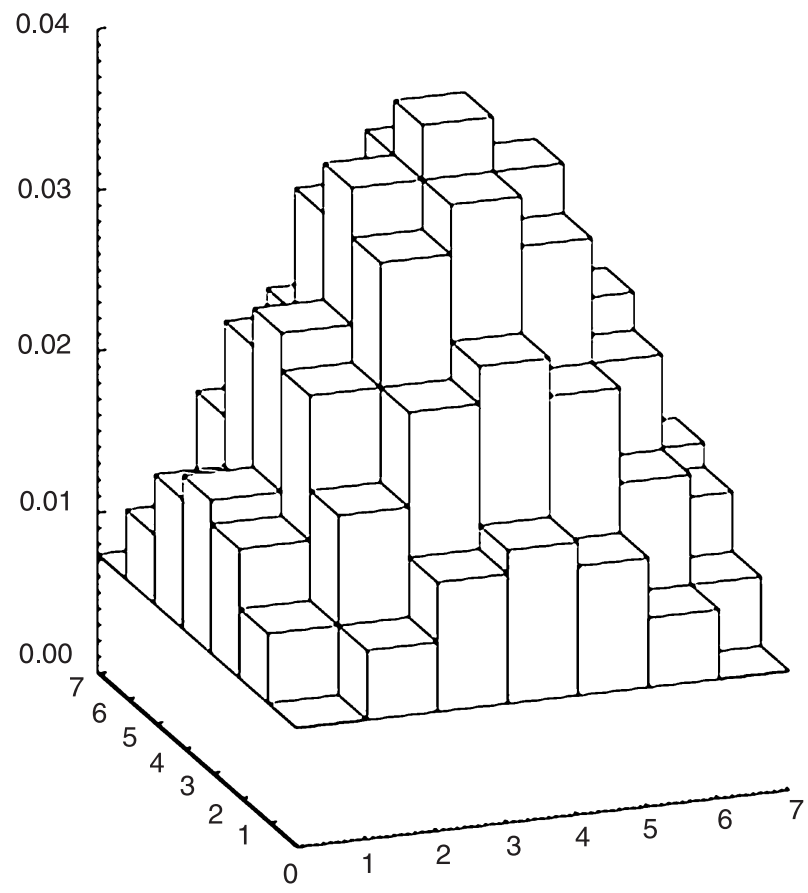

Fig. 5. 2-D Gaussian filter

$\vec{V}$. We use a bilinear interpolation to sample the value of $A^{\prime}$ from $A$. The new $A^{\prime}$ now replaces the original image $A$ and is used as starting point for the matching at the next lower pyramid level. Resulting disparities from each level are added to $\vec{V}$ until the full resolution image is matched (Fig. 3e).

With this pyramidal structure the "search radius" is given by at least $2^{(N+2)}$ pixels (corner to corner size is even $\sqrt{2} \cdot 2^{(N+2)}$ ) and therefore related to the highest pyramid level. With four pyramid levels $(N=3)$ a shift of at least 32 pixels can be detected. For mid latitude MSG infrared and water vapour images with $15 \mathrm{~min}$ time difference, this is equivalent to a cloud or atmospheric motion with more than $360 \mathrm{~km} / \mathrm{h}$. The search radius is also limited by the size of the original image, as edge effects occur if the kernel $\mathrm{Ke}$ is not fully covered by image data. The kernel used in this application of the matcher has a size of $7 \times 7$ pixel. At the third pyramid level it covers $56 \times 56$ pixels of the original image. Thus the detection of large displacements is limited at the outer approximately 50-80 pixels at each edge of the image depending on the direction of the motion. The image edges are therefore excluded from analysis for all following applications. Smaller ker- nels result in more blunders, i.e., false matches, larger kernels reduce the useful part of the image.

Result of the matcher is a disparity vector field $\vec{V}(\vec{P})$ and a warped image $A^{\prime}(\vec{P})$, which solves the optical flow problem in a scale dependent way. To be precise, this vector field is not a pure cloud pattern displacement field but also includes all local changes of cloud patterns as well (e.g., area changes). This has to be kept in mind for the following application.

\subsection{Image warping and morphing}

The warping of an image $A$ onto an image $B$ is given in Eq. (4). This technique can also be used for an interpolation that takes motion into account, the so called morphing. In order to get information on each pixel, not only the disparity vector fields $\vec{V}_{A \rightarrow B}$, mapping image $A$ onto $B$, but also the vector field $\vec{V}_{B \rightarrow A}$, which maps $B$ onto $A$ has to be taken into account. $\vec{V}_{B \rightarrow A}$ is similar but not identical to $-\vec{V}_{A \rightarrow B}$.

Using these vectors we calculate the interpolated image $\mathrm{D}$ at relative distance $\mathrm{d}$ from the first image as (e.g., $d$ is a relative time distance $0<t^{\prime} / \Delta t<1$, with $\Delta t$ as the time step between two satellite images):

$$
\begin{aligned}
D(\vec{P})= & d A\left(\vec{P}-d \vec{V}_{A \rightarrow B}\right) \\
& +(1-d) B\left(\vec{P}-(1-d) \vec{V}_{B \rightarrow A}\right) .
\end{aligned}
$$

With a factor $d>1$ a development can be extrapolated. While the interpolation by morphing is useful for the generation of additional satellite images between available time steps, e.g., of missing images, the extrapolation can be used for the nowcasting of the short range future of detected convective cell patterns.

\section{Detection}

Next step is the detection of the different stages of thunderstorm development. Different "development stages" are attributed to detections according to their significance for real thunderstorm activity - stage 1 - for strong local development of convective low level clouds, - stage 2 - for rapid cooling, or - stage 3 - for the detection of a mature cumulonimbus. 

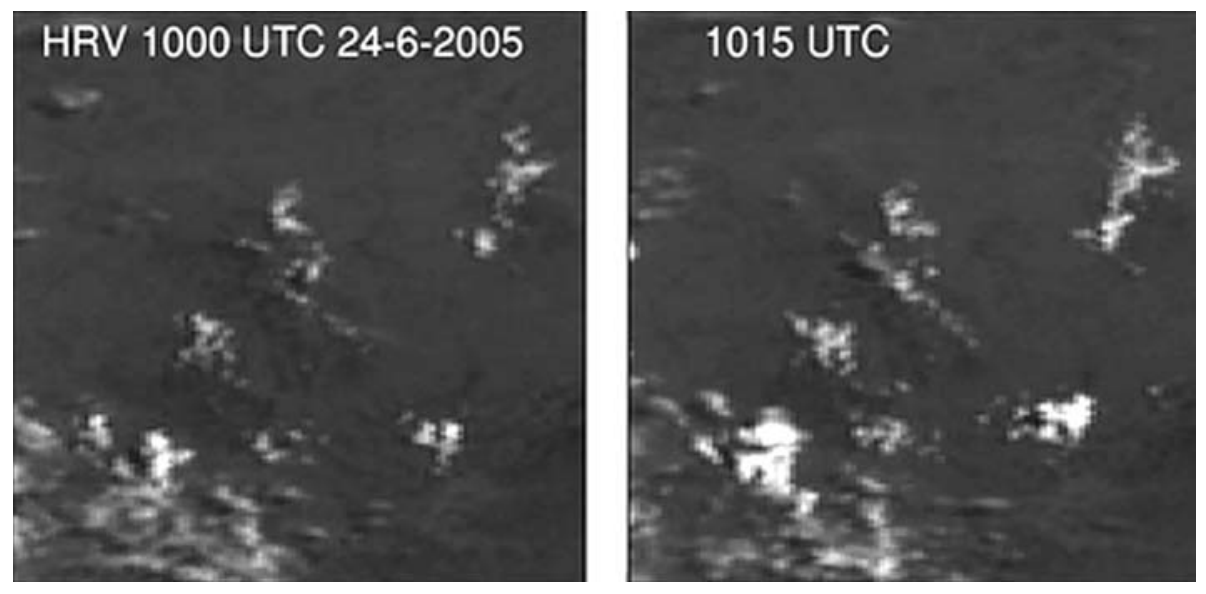

Fig. 6. 24 June 2005, cumulus fields over Central France exhibiting rapid development between the 10:00 and 10:15 UTC images

\subsection{Detection of convection initiation}

Early detection of low-level convergence often marked by shallow cumulus fields is supposed to be one of the clearest warning signals for the initiation of convection (Purdom 1976; Wilson and Mueller 1993; Wilson et al. 1998). With the SEVIRI HRV channel an unprecedented operational sensor is available for this purpose. A repeat cycle of only $15 \mathrm{~min}$ and a spatial resolution of about $1.5 \mathrm{~km} \times 1.5 \mathrm{~km}$ (for Central Europe) is ideal for the detection of rapid local scale development of the initiation of convection (see also Bugliaro and Mayer 2004). As not each detected cumulus humilis development will result in a mature cumulonimbus only rapid development is detected and a early stage 1 development is issued for such detections. Figure 6 shows an example of such a development within just 15 min.

The routine utilises a disparity vector field to get a first guess of the HRV cloud structures from the preceeding time step $t-1$ for the current time $t$ without any additional cloud area development. This first-guess image $\mathrm{HRV}_{\mathrm{fg}}$ (corresponding to $\mathrm{A}^{\prime}$ in Eq. (4)) is then subtracted from the current image at time $t$. The resulting difference field $\Delta \mathrm{HRV}=\mathrm{HRV}-\mathrm{HRV}_{\mathrm{fg}}$ reflects gains and losses in cloud area between the time $t-1$ and $t$ as positive and negative areas, respectively.

The selection of the right disparity vector field obtained with the matching algorithm (Sect. 2) is important, since these vector fields are not pure cloud motion fields, but also include changes of cloud amount. For example, the perfect reproduction of the HRV image at the current time $t$ would be obtained, if the disparity vector field
$\vec{V}$ extracted from the two HRV images at times $t-1$ and $t$ would be used. To avoid that, a vector field which does not contain the current visible image development itself, but still contains useful information on the cloud motion has to be used. Such a field can be generated by averaging over several time steps or by spatial averaging (to keep average motion on a greater scale) or by the use of an image pair from another spectral channel. Here a motion field is approximated by the disparity vector field derived from the IR images at the times $t-2$ and $t-1$ which, in addition, is smoothed by a running mean over $25 \mathrm{~km}$. This way an approximate motion field is obtained on a lower resolution that is independent from the local spatial and temporal developments to be analysed.

To further limit the detection to real low level cloud developments the following refinements are introduced: (1) Only HRV developments are taken into account which are supported by a cooling in the IR detected in an analogous way as the change in cloud area. Any positive cloud development has to be related to a cooling in the IR image indicating real cloud growth. (To avoid the above mentioned problems with local developments the difference field of $\Delta I R$ is obtained using WV images from time $t-1$ and $t-2$.) (2) Also excluded are regions which have a HRV reflectivity less than 0.5 . Thereby miss-detections due to moving fields of thin (dark) cirrus cloud fields over bright background (e.g., snow or lower cloud layers) are minimised.

Next the HRV and IR development information has to be combined. The application of separate thresholds to the quality fields $\triangle \mathrm{HRV}$ and $\Delta \mathrm{IR}$ would lead to a loss of the possibility of a 


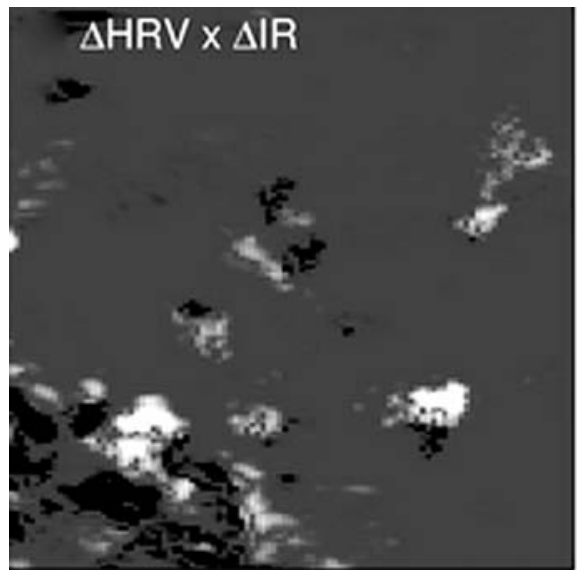

a

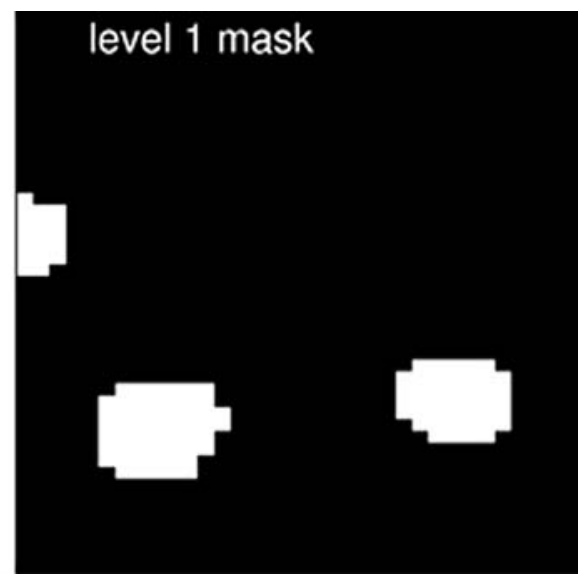

b
Fig. 7. Development stage 1, low cloud development, 24 June 2005: cumulus field over Central France at 10:00 and 10:15 UTC (cf. Fig. 6). Shown is the quality field $\triangle \mathrm{HRV} \times$ $\Delta \mathrm{IR}$ (a) reflecting the areas of cloud growth (bright) and cloud dissolution (dark). The cloud mask (b) marks the areas of clearest area growth weighted consideration of both criteria. Instead, we introduce a fuzzy logic combination (which is used repeatedly in the following). After a normalisation of the $\triangle \mathrm{HRV}$ and $\triangle \mathrm{IR}$ difference fields, a product of both fields is generated $-\Delta \mathrm{HRV} \times \Delta \mathrm{IR}$. Thereby a quality field is provided showing areas of growth in cloud amount, inferred from HRV brightening and IR cooling (Fig. 7a). Larger values indicate either a remarkable development in one of the channels or a less prominent development unambiguously detectable in both channels. Areas with values above a certain threshold are selected and stored as a cloud mask (Fig. 7b). The threshold value corresponds to features of about 3 times the size of typical standard deviations in the fields $\triangle H R V$ and $\triangle I R$. Finally, each pixel detected at this detection step is assigned a development stage 1 .

\subsection{Detection of rapid cooling}

The stage 2 is issued for rapid vertical developments detectable in the thermal spectral range. The WV channel is chosen as upper tropospheric developments are emphasised in this channel. Lower tropospheric cooling (growth) is covered by development stage 1 . The only cloud features clearly detectable in this channel are specifically high cloud tops and their rapid cooling detectable in consecutive $\mathrm{WV}$ images is most likely caused by rapid growth of severe convective cells.

The development detection is based on the same principles as before - a first guess water vapour image is extrapolated and subtracted from the current WV image to obtain $\Delta \mathrm{WV}$. The disparity field used for this purpose is extracted from the time steps $t-2$ and $t-1$. As before this choice is due to the need to use a vector field representative of advective motion in the level of interest, but free of local developments (IR or VIS data would not reflect upper tropospheric motion, time steps $t-1$ and $t$ would contain the local development to be analysed). Again a threshold difference value (defined as above) is selected to find the areas of intense not advection-related developments (Fig. 8). The development stage assigned to these areas is 2. Cell patterns detected at this stage are already quite likely to have a hazardous intensity.

\subsection{Detection of mature thunderstorm cells}

Opposed to the two levels of early development stage 1 and rapid cooling stage 2 which are optimised in a way to be rather sensitive in marking potential cells of severe convection the main interest in the following is the accurate detection of mature convective cell patterns as well as the detection of active cell centres after a characteristic cirrus anvil has been developed. The detection of active convective centres of mature cumulonimbus is bound to areas exhibiting a WV temperature close to or smaller than the temperature of the current tropopause in combination with a large local gradient of reflectivity in the HRV channel.

This is similar to the temperature threshold criteria in other satellite data based tracking algorithms (ranging from 218-245 K); e.g., Arnaud et al. (1992); Machado et al. (1998); Carvalho 


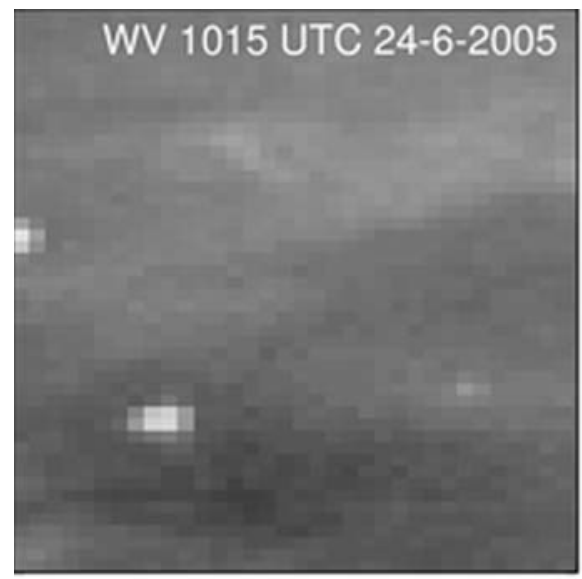

a

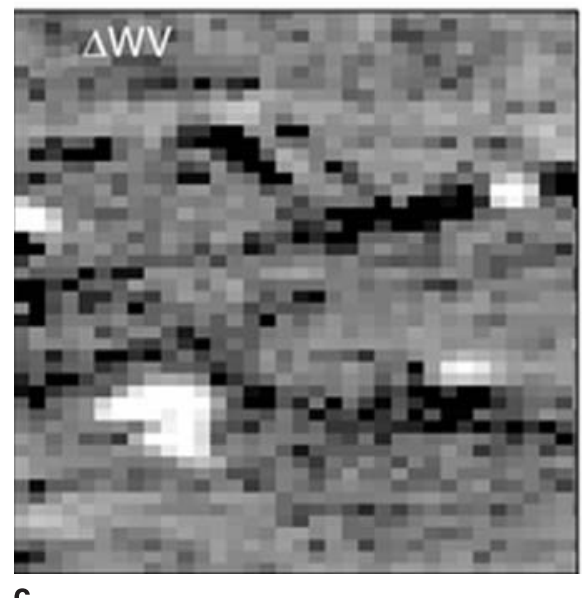

C

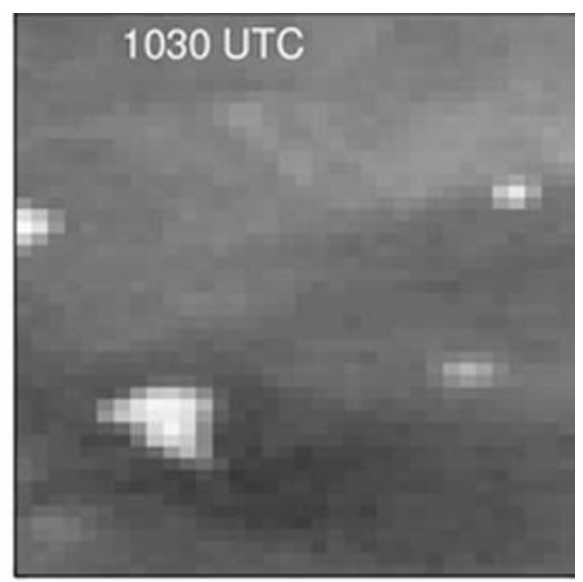

b

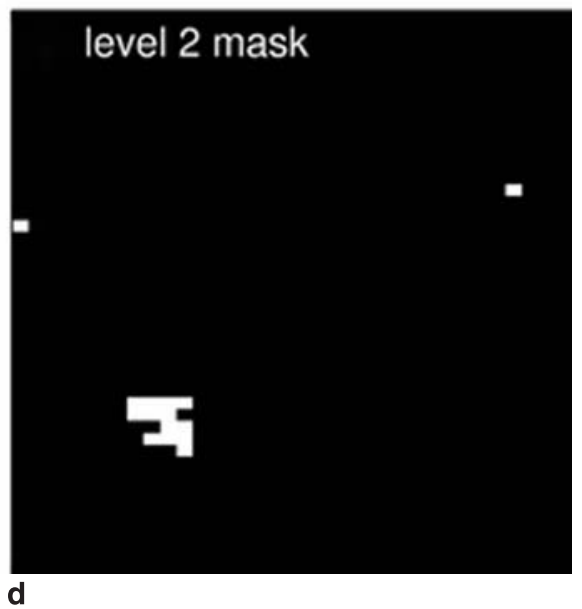

Fig. 8. Development stage 2, i.e., rapid cooling, for 24 June 2005: cumulus field over Central France $15 \mathrm{~min}$ after the situation displayed in Fig. 6. A cooling is detectable from the WV images $(\mathbf{a}, \mathbf{b})$. The difference field $\Delta \mathrm{WV}$ (c) reflects the cooling (bright) and warming areas (dark). The mask (d) selects the areas of maximum cooling and Jones (2001); Morel and Senesi (2002) apart from three important differences:

(1) The WV is used instead of the IR as the WV image clearly indicates severe convective cells as especially cold overshooting features above the tropospheric water vapour background.

(2) Not a fixed but an adaptive temperature threshold is used which allows for a consideration of the synoptic situation as well as seasonal differences in typical convective cloud top temperatures.

(3) The local variability of the HRV reflectivity is used to further refine the detection of the active centres of cell patterns.

For the second point the current temperature of the tropopause determined from ECMWF model analyses is used (ECMWF, 2005, MARS Archive). The analysis is provided daily at 0,6 , 12, and 18 UTC. Here lapse rate tropopause tem- perature (World Meteorological Organisation 1957) is used and is determined from the atmospheric profiles available in the region of interest. The algorithm described in Sect. 2.2 is used to best interpolate the tropopause temperature field for each image time. If Cb-TRAM is used in realtime nowcasting situations, short range forecast data is used instead of the analysis data.

The temperature threshold linked to the lapse rate tropopause temperature $T_{\text {trop }}$ is chosen to be $\mathrm{T}_{\text {trop }}-1.5 \mathrm{~K}$. This value representative of clouds reaching a height slightly below the actual model tropopause is found by extensive testing to work best both for isolated air mass thunderstorms and along frontal systems with abrupt changes of tropopause height. The temperature criterion is now to be combined with the secondary criterion for the detection of active cell patterns, the HRV roughness. A quantitative combination with this second criterion is again reached using fuzzy logic. A quality field is generated from the 


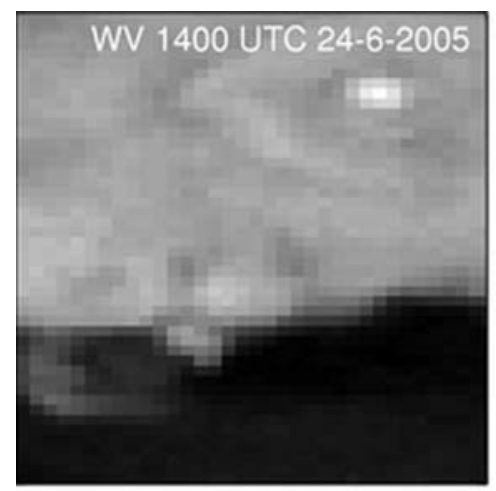

a

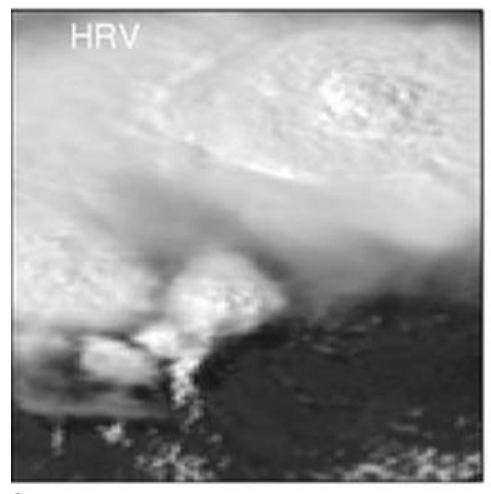

C

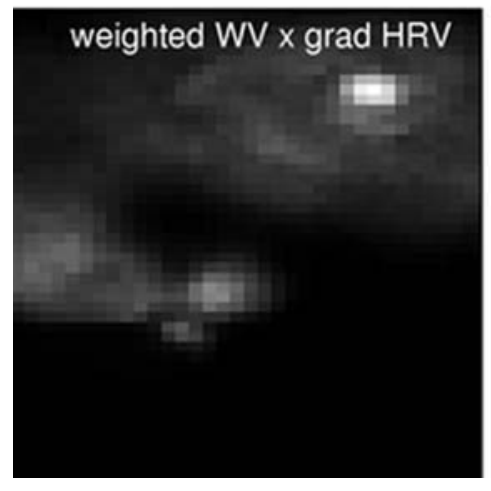

e

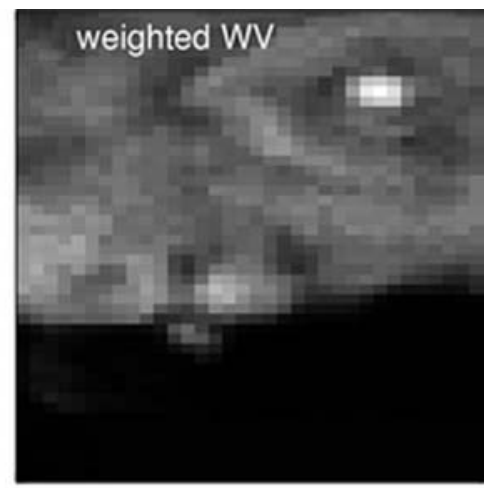

b

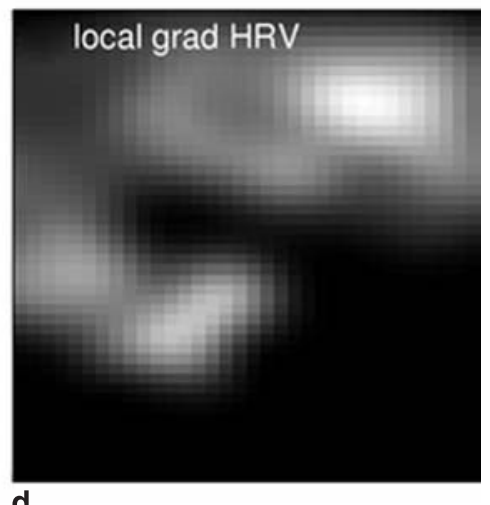

d

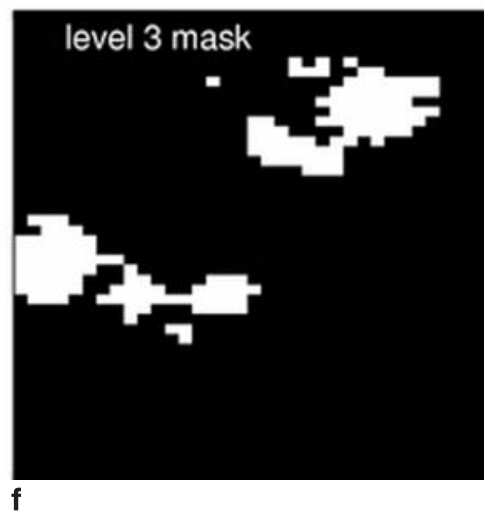

Fig. 9. Development stage 3, i.e., mature convective cell patterns, for 24 June 2005: shown is the same region over Central France (cf. Figs. 6-8) at 14:00 UTC. Extracted from the WV (a) and HRV images (c) are the quality fields "weighted WV" (b) and "HRV roughness" (d). These are then combined by multiplication (e), and finally a threshold is used to mark the areas of clearest convective activity
WV temperature field using the hyperbolic tangent function to spread the value range around the threshold without completely loosing the information detail below the threshold (compare Fig. 9a and b).

Figures 1a and 9c show the characteristic HRV roughness. The regions of strongest up-draft are not only marked by the highest and thus coldest cloud tops, but even more generally by the strongest variability in cloud top height. This variability leads to strong geometric illumination effects (shadows and bright slopes) generating visible roughness, which is particularly well detectable in the HRV. The measure of variability is a two- dimensional gradient value obtained for the local surrounding of each pixel of the field.

This secondary criterion facilitates the separation of rough up-draft from smooth outflow regions at the top of thunderstorms or larger mesoscale frontal cloud systems, and at the same time it allows for the detection of clouds which might still be slightly below the temperature threshold, but show a distinct variability of the cloud top. The local gradient field is strongly depending on illumination, and thus it is normalised with the typical diurnal variation of the local HRV gradient (obtained from the analysis of a number of days for Central Europe) before the 


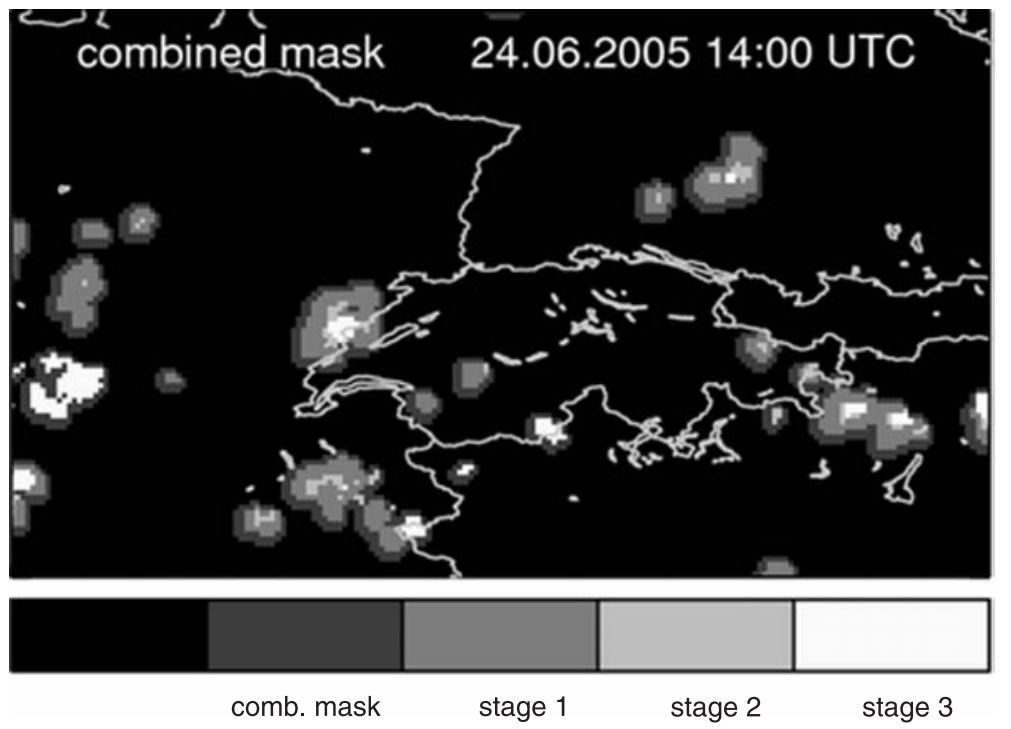

Fig. 10. Combination of development stage masks for the situation shown in Fig. 1: Three stage sub-masks are combined, patterns smaller than 2 pixels are filtered, and the remaining cell patterns are extended to the final combined mask two quality fields are combined by multiplication. A threshold is set to select the cloud cell patterns of interest (Fig. 9e and f). These cloud areas correspond to active convective cells which are either distinctively high (cold), display a strong cloud top turbulence (strong up-drafts), or both. The development stage of 3 for mature thunderstorm is assigned to the detected cloudy pixels.

When HRV reflectivities are no longer available at sunset, the detection of initial cumulus development (Sect. 3.1) is de-activated and the detection of mature thunderstorms (Sect. 3.3) is adjusted in a way that only the part using WV temperature is used and the best possible transition between day and night mask is achieved.

As mentioned before, thresholds used throughout the detection of stage 1-3 are optimised by testing with a data set from a number of convective events throughout continental Europe. Values were not always given since the fuzzy logic combination of quality fields often leads to a loss of easily understandable physical meaning of values and thus does not add clarity to the description.

\subsection{Combination of development stage masks}

The three development stage masks (Figs. 7b, 8d, and 9f) are combined to one mask displaying all the detected cell patterns which are to be tracked in the following (Fig. 10). If a cell pattern contains detections of different development stages, the most developed stage is chosen to characterise the whole pattern. To be accepted for tracking detected cell patterns must have a minimum area of more than two connected normal resolution SEVIRI pixels, i.e., about $50 \mathrm{~km}^{2}$. Individual cell patterns closer than about $10 \mathrm{~km}$ (2 SEVIRI pixels) are merged as large patterns are easier to track - at the same time the discrimination of individual cells is somewhat blurred this way.

\section{Tracking}

\subsection{Basic algorithm}

In general, the tracking of detected cell patterns, whether radar or satellite data based, is divided into two main techniques: pattern-oriented correlation techniques and overlapping techniques. The technique widely used in the determination of general cloud motion vectors is the pattern oriented correlation technique. An arbitrary (in some respect prominent) cloud pattern in one image is recovered in the next one, by finding the position of maximum correlation between the identified pattern and a part of the new image. Several applications of this principle can be found for different tracking algorithms, e.g., Schmetz et al. (1993), Mecklenburg et al. (2000), or Bolliger at al. (2003). This method even works for images separated in time for one or more hours (Carvalho and Jones 2001).

The second - the overlapping - tracking method relies on a short image repeat cycle. It is based on the overlap of identified cell patterns in two consecutive images and thus only works as long 
as overlap occurs, which depends on the time interval between two images and on the size of the tracked cell patterns. An important improvement to this method is the use of the approximate moving direction and velocity of a detected cloud pattern at time $t-1$ to retrieve a first-guess of position and size of the pattern at time $t$. This way better overlap with the new (time $t$ ) cell pattern detections is given. Examples of application are given in Arnaud et al. (1992), Dixon and Wiener (1993), Morel and Senesi (2002), or Handwerker (2002).

The Cb-TRAM algorithm utilises the latter method. The minimum cell pattern size of $50 \mathrm{~km}^{2}$ is exceptionally small for satellite tracking algorithms compared to most of the hitherto existing algorithms and therefore requires (a) a high time resolution, which is provided by the SEVIRI sensor (15 min), and (b) a detailed approximation of the cloud motion field to find overlap between two consecutive images, which is provided by the pyramid matching algorithm (Sect. 2). Where other tracking algorithms are reliant on average wind vectors derived for the whole image or from single cell's history, our algorithm uses an approximate motion field, defined for each image pixel. That way not only the displacement but also trends of area or shape changes are considered.

Starting from the combined cell pattern mask (Sect. 3.4) at time $t-1$ a first-guess cell pattern mask is extrapolated for time $t$ by application of Eq. (4) with the disparity vector field derive from
WV images. For each pattern detected at time $t$ the maximum overlap with the extrapolated cell patterns detected at time $t-1$ is determined (Fig. 11a). If no overlap is found a new cell pattern number is issued which will be used throughout the whole tracking cycle to unambiguously identify the cloud pattern. A history log file is created for each cell pattern, which is also used by the displaying routine later. At each time step $t$ the detection time, the detected pattern's pixel positions, the development stage, the position of the centre of gravity (weighted with 1$\mathrm{T}_{\mathrm{IR}}$ ), and the displacement since last time step $t-1$ are stored for each pattern detected. If overlap is found for several cell patterns the number of the pattern with the maximum overlap is identified and the related history log file is updated. If a cell pattern at time $t$ overlaps with more than one of the extrapolated patterns of time $t-1$ only the track of the pattern with the largest overlap area is continued. The other cell patterns die, a cell merging has happened. If more than one cell pattern at time $t$ overlaps with an extrapolated pattern from time $t-1$, the one with the largest overlap area inherits the history. For all other patterns new history logs are initialised, the cell splits.

\subsection{Monitoring of cells no longer detected}

The disparity vector fields extracted following Sect. 2 were already extensively used for the detection of cloud developments before. Of course,

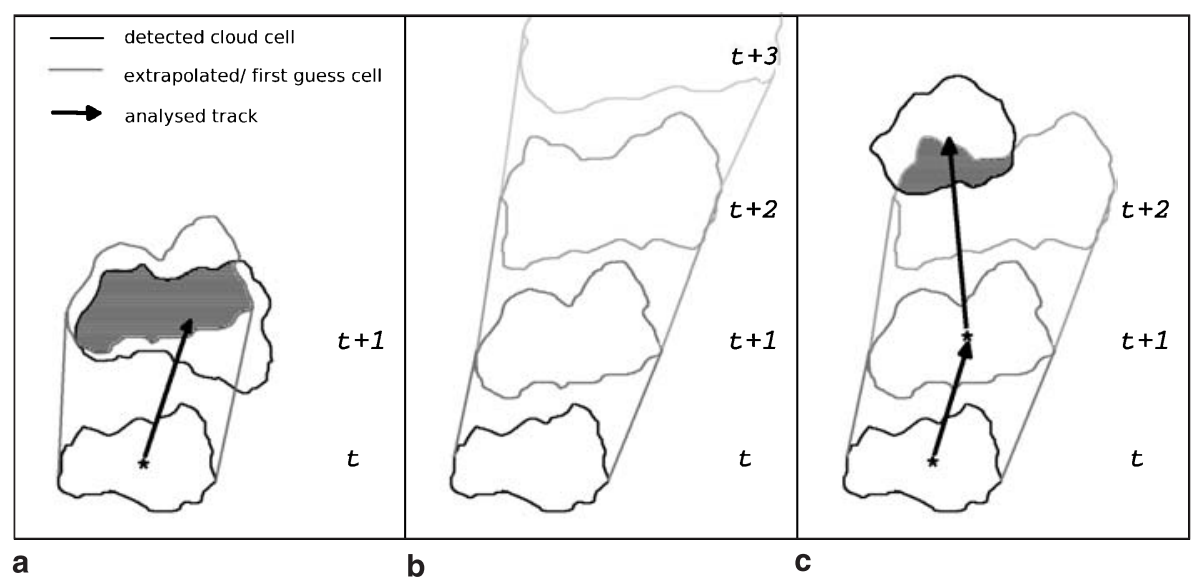

Fig. 11a. Basic tracking principle: a cell pattern detected at time $t$ is recovered at time $t+1$ through determination of the overlap using the first guess extrapolation; (b) extrapolation: forecasts of the patterns shape for the following time steps are obtained by extrapolation using the differential disparity vector field; (c) monitoring of patterns no longer detected: the extrapolated patterns are used to bridge gaps in detection 
these fields can be used for short range extrapolations as well (Fig. 11b). In contrast to the extrapolations used in the detection of rapid developments (stages 1 and 2) this time an extrapolation is sought which provides not only advection, but the local developments as well to get the best possible guess for a future detailed shape of a cell pattern. Consequently, extrapolations are generated using the most recent IR disparity vector fields on a pixel by pixel basis. IR data is preferred for this task since it offers detailed informations for clouds in all vertical levels. For up to 4 time steps $(1 \mathrm{~h})$ the predicted cell patterns are stored in the history log files. Not only the current velocity but also the current stage of the convective life-cycle is extrapolated. For example, a detection of a thunderstorm cell growing during the last $15 \mathrm{~min}$ interval will result in a prediction of further growth for the next time steps.

Sometimes the tracking for particular cell patterns, especially during the development stages 1 and 2 , is interrupted for short time intervals, because phases of weak cloud development are not detected. For example, a early development stage 1 is issued because of rapid low level cumulus development at a time $t$. After this initial stage no further development is detectable that is strong enough to meet one of the detection criteria, because there is only a slow vertical growth of the cell without further horizontal extension. Nonetheless, $30 \mathrm{~min}$ later the convective development becomes more intense and the rapid cooling stage 2 is triggered. Without an additional monitoring of cell patterns once detected, the stage 1 pattern at time $t$ would die out and a new one would be initialised at time $t+30 \mathrm{~min}$. The different phases of the cell development would be disconnected. Therefore, the tracking is continued after the last detection for two more time steps. A synthetic cell pattern is introduced into the tracking mask using the extrapolation patterns (Fig. 11c). Only if cell patterns are not detected again within $45 \mathrm{~min}$, the tracking of a specific cloud pattern ends.

\section{Comparison with radar and lightning data}

Figure 12 shows examples of the Cb-TRAM displaying routine products. A SEVIRI HRV satellite image (IR, WV also selectable) and a map that gives country borders, lakes, rivers, and coastlines are overlaid. The three development stages are marked by different colours: "Yellow" marks stage 1 detections, "orange" stage 2 and "red" stage 3 . The edge of cell patterns currently detected can be depicted by a line, their centreof-gravity by a star (Fig. 12b). The pattern's history is represented by the track connecting the successive positions of the centre-of-gravity. The part of the track showing the displacement of the cell pattern during the preceeding $15 \mathrm{~min}$ is coloured according to the current development stage. Times noted on the map give the time of first detection of each cloud pattern. The green colour represents the monitoring of those cell patterns that are not detected at the current time but at least twice during the last $60 \mathrm{~min}$. For these patterns the extrapolated displacement and the assumed position of the centre-of-gravity is depicted. In different tones of grey the extrapolated future extent and position of the cell patterns can be displayed for $15,30,45$ and 60 min into the future for all patterns that have at least reached stage 2 (Fig. 12a).

In order to test the ability of Cb-TRAM to detect thunderstorm clouds, radar and lightning data are certainly apt independent data source for comparison. Such data allows to distinguish between small convective clouds and real thunderstorm cells by the selection of certain levels of radar reflectivity and the occurrence or absence of lightning. A typical characterisation of precipitation (or respective thunderstorm) intensity in terms of radar reflectivity, e.g., the one used by the US National Weather Service, is given as follows: Reflectivity levels between 38 and $44 \mathrm{dBZ}$ are indicative of moderate to heavy rain, 44-50 dBZ denote heavy rain, 50-57 dBZ very heavy rain with hail possible, and more than $57 \mathrm{dBZ}$ very heavy rain with large hail possible. Lightning frequency has been found to be proportional to the intensity of the up-draft velocity (Baker et al. 1995) and the collision and growth of ice particles (Takahashi 1978). Therefore, within convective cell patterns detected by $\mathrm{Cb}$ TRAM we should find both radar and lightning signals of an intensity dependent on the level of convective activity and maturity of the cells. For the comparison we selected two different weather situations, already shown in Fig. 12, the 24 June 2005 and the 12 August 2004. The first is 


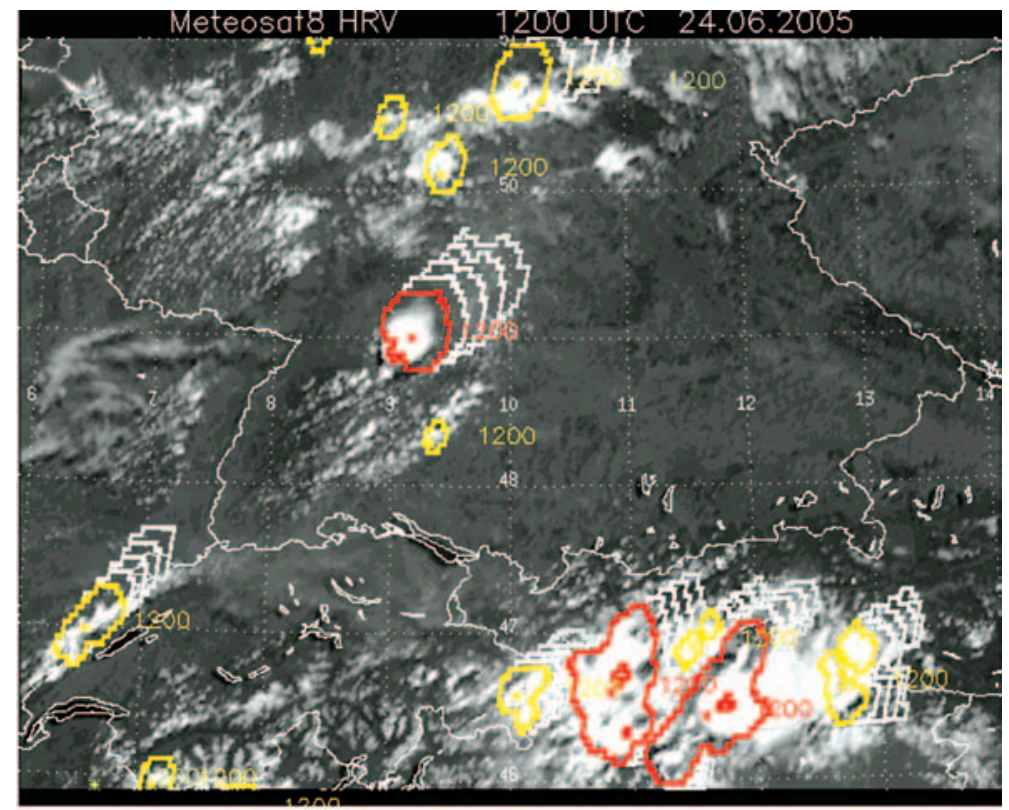

a

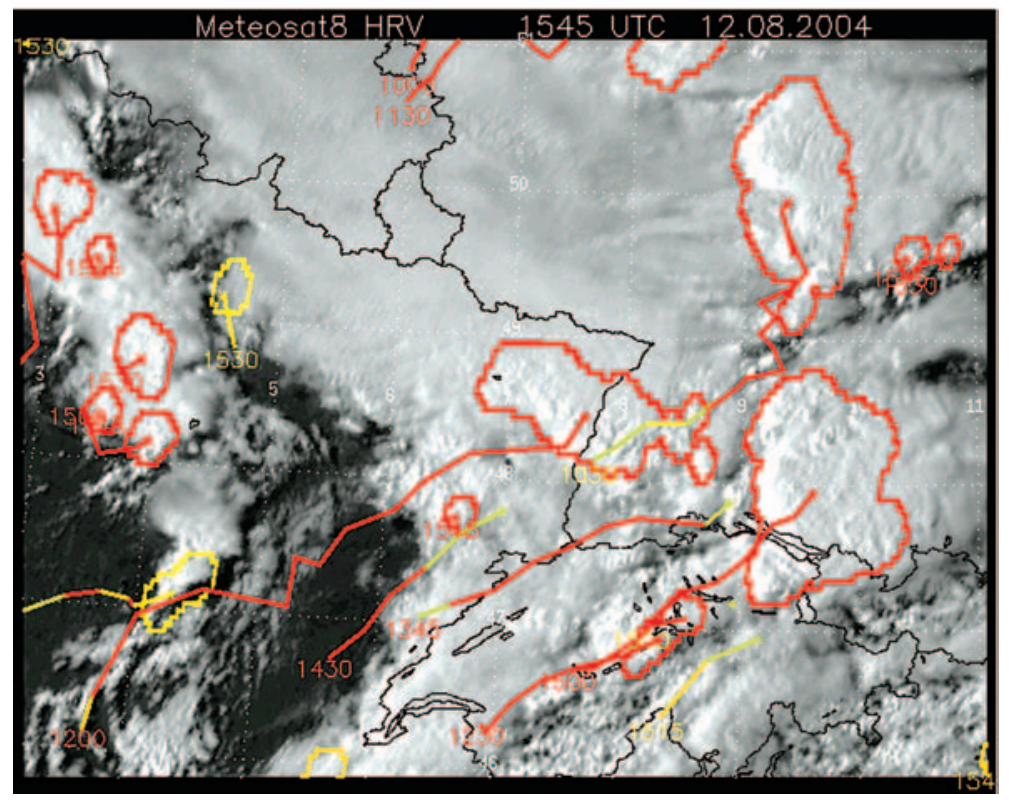

b
Fig. 12. Examples of Cb-TRAM application for nowcasting and tracking: (a) detection and forecast of air mass thunderstorms over Southern Germany and the Alps at the 24 June 2005 12:00 UTC (compare Fig. 1); (b) detection and tracks of thunderstorms evolving ahead and behind a cold front extending from Switzerland in the South over Eastern France and the Low Countries to the North at 12 August 2004 15:45 UTC. For details on lines and colour coding please see text a weather situation with generally unstable summertime conditions and weak synoptic forcing with consecutive development of individual thunderstorm cells during the day (Fig. 12a). The second case is dominated by the passage of a cold front through middle Europe accompanied by a pre-frontal thunderstorm line and a postfrontal convection line (Fig. 12b).

In Fig. 13a, detected clouds from Cb-TRAM are overlaid with radar reflectivity from the European radar composite of DWD (German
Weather Service), in Fig. 13b they are overlaid on the WV image including the detected lightning flash positions. The radar reflectivity provided by the European composite is divided into similar reflectivity bins as the classification mentioned above. Little patches of coloured shading indicating echo levels greater than $28 \mathrm{dBZ}$ (green, moderate rain), $37 \mathrm{dBZ}$ (moderate to heavy rain) and greater $46 \mathrm{dBZ}$ (dark blue, heavy rain) are depicted in Fig. 13a. Smaller reflectivity values are not shown as only high values are an 


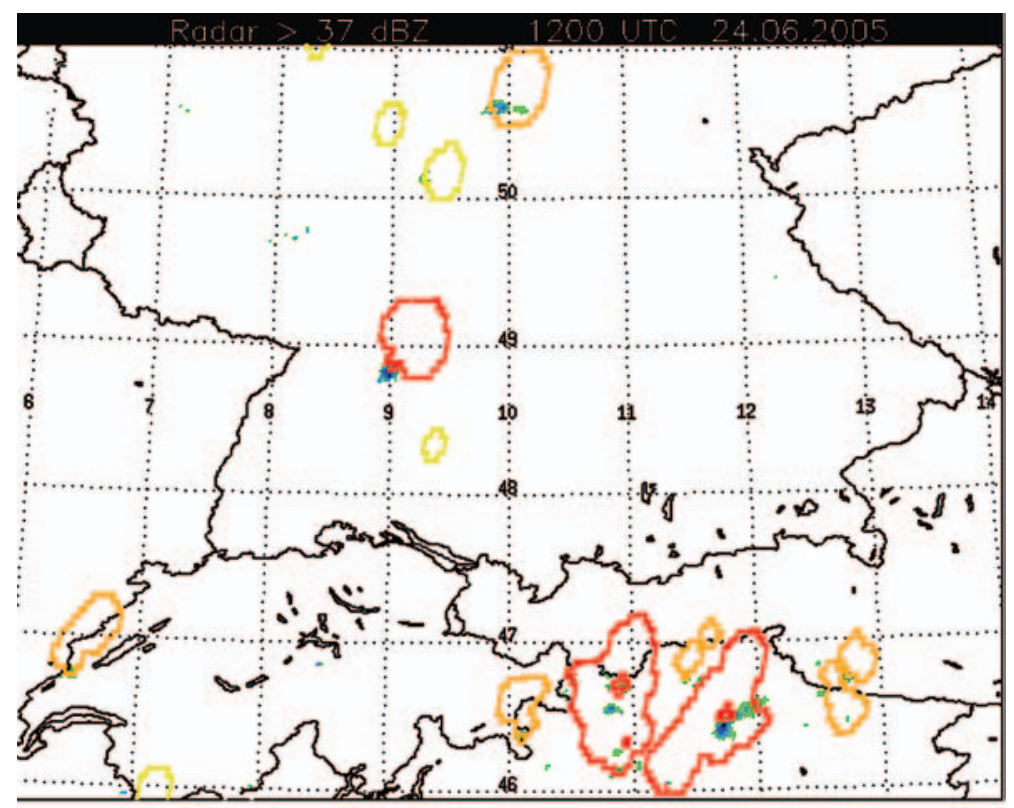

a

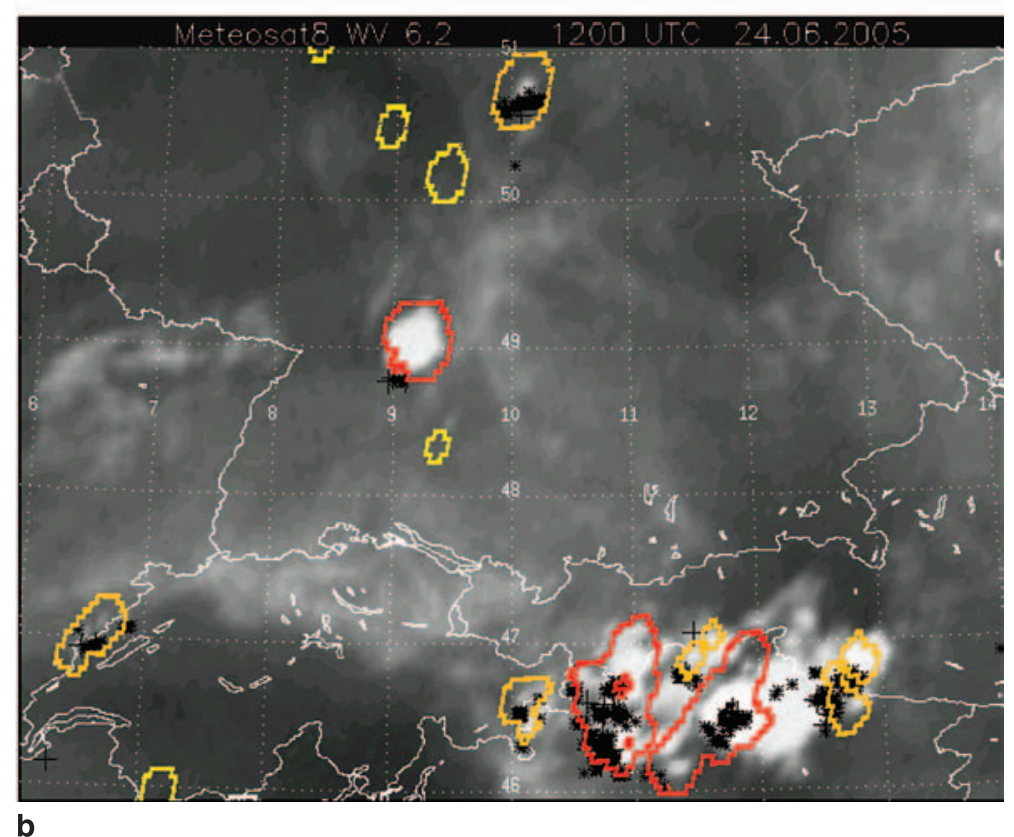

Fig. 13. Case 24 June 2005 12:00 UTC: overlay of identified clouds from Cb-TRAM (a) with radar reflectivity $(>28 \mathrm{dBZ}$, moderate rain, green; $>37 \mathrm{dBZ}$, moderate to heavy rain, light blue; $>46 \mathrm{dBZ}$, heavy rain, dark blue) and (b) with detected lightning activity within the last $15 \mathrm{~min}$ (positive ground to cloud flashes marked by + , all other marked by $*)$. In $(\mathbf{b})$, the WV image is underlaid as background evidence of thunderstorm activity (and for reasons of clarity). Coloured contours in Figs. 13 and 14 are identical to the respective contours in Fig. 12. It can be seen that within the red bounded regions, i.e., the regions of development stage 3 (mature cumulonimbus), spots of high radar reflectivity as well as the majority of lightning detections can be found. Only for the cell pattern in southern Germany, at about $9^{\circ} \mathrm{E}$ and $49^{\circ} \mathrm{N}$, the patch of high reflectivity and lightning activity is just at the edge of the detected cell pattern at its southwestern end. Another patched of a radar reflectivity greater than $37 \mathrm{dBZ}$ and lightning activity is visible within the $\mathrm{Cb}$ TRAM cell pattern at $10^{\circ} \mathrm{E}, 50.5^{\circ} \mathrm{N}$. This cell pattern is marked as a region of development stage 2, i.e., rapid cooling (orange contour in Fig. 12a). It is notable that high radar reflectivities are neither found within the patterns of early development stage 1 (yellow contour) nor within the other patterns of stage 2 over the Alps and at the French/Swiss border in the lower left corner 
of the figure. On the contrary lightning is found in some of the stage 2 cell patterns.

Summarising, for this scene Cb-TRAM seems to be capable of correctly identifying the mature thunderstorm cells in the sense that cell patterns assigned the development stage 3 do exhibit prominent convective activity. Considering the additional information that the stage 1 patterns at $9.5^{\circ} \mathrm{E}, 48.3^{\circ} \mathrm{N}$ and $6.5^{\circ} \mathrm{E}, 47^{\circ} \mathrm{N}$ do both in fact reach the mature thunderstorm stage 3 at 12:45 UTC, also the onset of convection before precipitation or lightning occurs is correctly detected. In between those two stages the development stage 2 includes patterns which exhibit less severe or less definite thunderstorm activity, e.g., the cell patterns without clear precipitation radar echo but already showing lightning activity.

The inspection of Fig. 12b showing the second case reveals a more complex cloud situation. The broad white band covering the centre of the image is a frontal zone, predominantly showing a smooth cloud structure of high cirrus. Ahead of

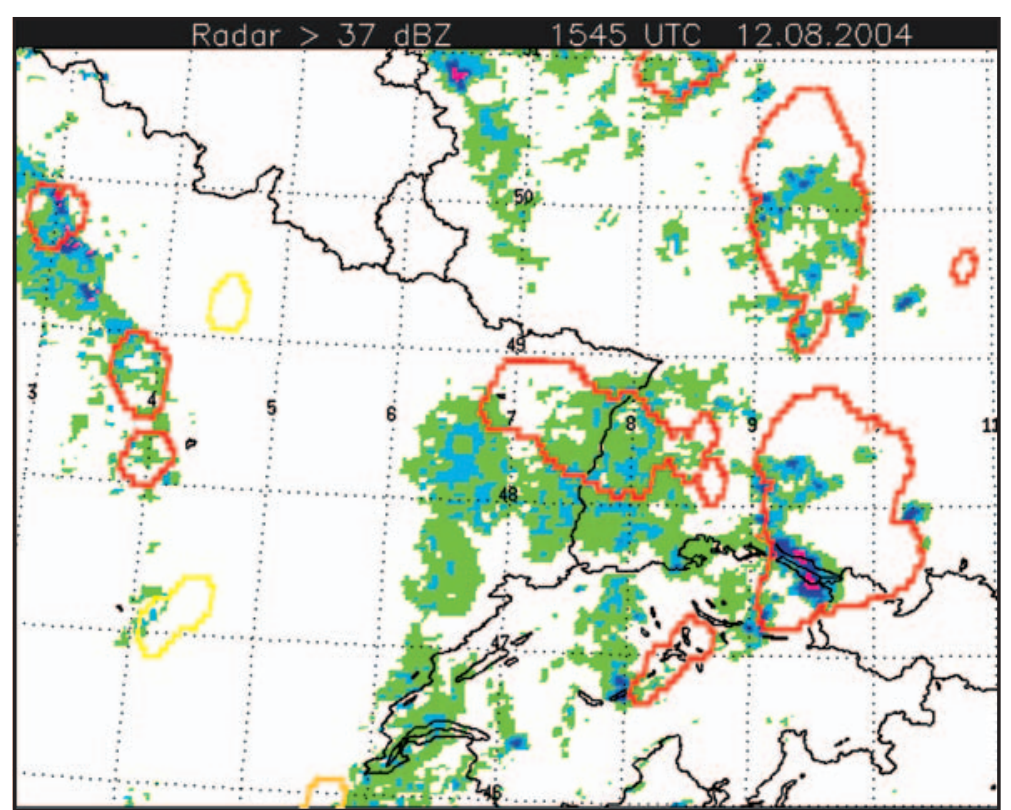

a

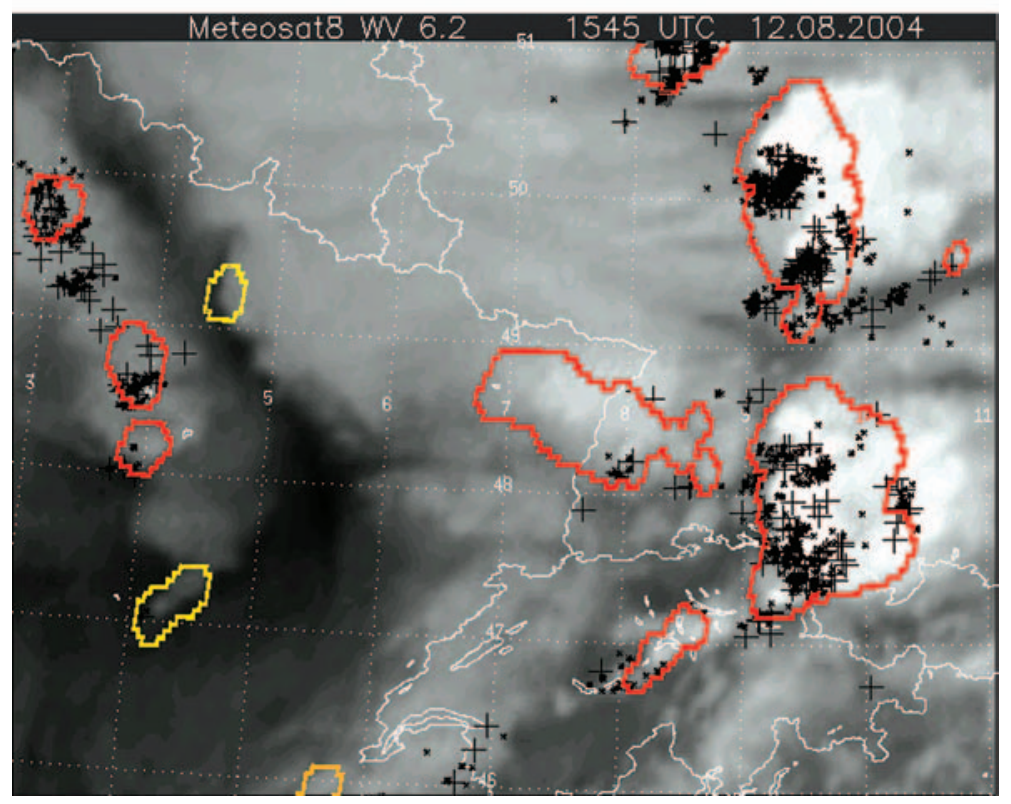

b
Fig. 14. Case 12 August 2004 11:45 UTC: overlay of identified clouds from Cb-TRAM (a) with radar reflectivity ( $>28 \mathrm{dBZ}$, moderate rain, green; $>37 \mathrm{dBZ}$, moderate to heavy rain, light blue; $>46 \mathrm{dBZ}$, heavy rain, dark blue; $>55 \mathrm{dBZ}$, very heavy rain with hail, purple) and (b) with detected lightning within the last $15 \mathrm{~min}$ (positive ground to cloud flashes marked by + , all other marked by $*)$. In $(\mathbf{b})$, the WV image is underlaid as a background 
the front to the East the pre-frontal thunderstorm cell patterns are marked by red contours. Some cell patterns of development stage 3 are also marked behind the cold front within a convective line over France. Furthermore, a large stage 3 pattern is situated well within the frontal zone (centred at about $7.7^{\circ} \mathrm{E}$ and $48.5^{\circ} \mathrm{N}$ ). Referring to the overlay with radar and lightning (Fig. 14), clear signs of heavy rain (in the pre-frontal cells reaching the hail level) and strong lightning activity are visible for the stage 3 patterns ahead and behind the front. Notably within the frontal zone no lightning flashes are detectable besides the few flashes in the region close to the stage 3 cell pattern identified by Cb-TRAM. However, there are areas of moderate radar reflectivities visible at some locations outside the identified patterns.

Of course, strong precipitation along a summertime cold front is a common process, even without severe thunderstorm activity and these cases of advective precipitation are not supposed to be detected by Cb-TRAM. With respect to the convection line behind the front it has to be emphasised that its detection together with the pre-frontal cells is only possible due to the adaptive tropopause temperature criterion. Since the tropopause temperature increases strongly as the front passes through, a fixed thunderstorm top temperature threshold would either provide no detection of the post-frontal cells or provide a very indiscriminate detection of the whole frontal cloud cover. Still, at least one individual cell in the post-frontal convection line is missed. However, Cb-TRAM should not be expected to resolve any individual cell within a thunderstorm line especially when these features are of smaller scale, due to the limited information content analysing cloud (mainly cloud top) characteristics from a satellite sensor. It can be seen that $\mathrm{Cb}$ TRAM is able to separate thunderstorm cells from frontal cirrus cloud systems of similar brightness temperature and to some extent the active cell centres from thunderstorm cirrus shields.

In fact, there seems to exist one striking detection failure of the Cb-TRAM diagnosis in this case. That is the small radar reflectivity cell with purple shading (greater $55 \mathrm{dBZ}$ ) at about $6.3^{\circ} \mathrm{E}$, $50.7^{\circ} \mathrm{N}$. This feature within the frontal line has been detected within the preceeding $2 \mathrm{~h}$ as well, however without any detectable indication in the satellite images (cf. the HRV image in Fig. 12b and the WV image in Fig. 14b). On the one hand, the radar data might not give the true picture of precipitation processes due to incomplete radar coverage, beam blocking by orography and different scanning modes of the radars within the radar network providing the composite. On the other hand, the strong precipitation there is obviously not produced by a thunderstorm as it should be apparent in the IR and WV images then.

Concluding for these two cases of thunderstorm development and propagation, Cb-TRAM is not only capable of correctly identifying thunderstorm cells but also of the detection of several stages of the convective life-cycle including the early onset of convection at a time when corresponding signals in radar and lightning are not yet visible.

\section{Conclusion}

In this paper, a new automated tool for the detection, tracking and nowcasting of thunderstorm cells was presented which is based on information from three different channels of the Meteosat-8/SEVIRI sensor and on ECMWF operational analysis data. Detected are three different combinations of characteristics related to three stages of the convective life-cycle. Each of them is particularly interesting be it that it allows for an early issue of warnings of potential convective activity or that it marks fully developed severe convective activity. Especially the two development stages for rapid convective developments, which use two consecutive satellite images in the HRV, IR, or WV channels, represents a novel exploitation of the specific possibilities offered by the SEVIRI sensor - short image repeat cycle and high resolution information (HRV). The traditional method of detection of mature severe convection, a temperature threshold in an infra-red channel, is extended to more generality by the use of ECMWF tropopause information. That way, the detection automatically adapts to variable seasonal or current weather conditions. During day-time the availability of the HRV is used to further restrict the detection of mature cells to the most active cell centres by the analysis of high resolution variability.

The central techniques of the tracking algorithm linking detected cell patterns in consecu- 
tive images are the pyramid matching algorithm and the analysis of overlap between detected cell patterns in two time steps. The former provides disparity vector fields describing the development of patterns during a time step. The latter looks for spatial overlap between detected patterns in one time step and extrapolations of the preceeding time step's detected cell patterns. The disparity vectors provide the basis for an interpolation of satellite images or other data fields for intermediate time steps between two known data fields as well as for short-range forecasts of developments by extrapolation.

Two cases - thunderstorms embedded in a mesoscale frontal system and isolated air mass thunderstorms - were presented which illustrate the capabilities of the new algorithm. The detection algorithm was tested by comparison of detected convective cell patterns to precipitation radar echoes and lightning data from operational networks.

\section{Discussion}

From this point further more systematic verification is to be conducted, e.g., through the analysis of conventional error measures or preferably through the use of object-oriented verification procedures (e.g., Ebert and McBride 2000). Conceivable are the extension of the early development detection capabilities through satellite data based determination of air mass stability (as discussed in Senesi et al. 1998) or the identification of active cell centres with precipitation radar data or lightning networks.

Nonetheless, the demonstration case section of this paper widely supports the used detection approach. Maxima of precipitation and lightning activity were always found within the identified cell pattern outlines although the detected patterns tend to include areas of low convective activity as well. On the one hand, this of course represents a general shortcoming of satellite data based detection as only secondary effects of severe convection (cloud top temperature or cloud top turbulence) can be evaluated. On the other hand, the fact that not every detected area includes clear signals of heavy precipitation or lightning activity - mainly the cell patterns with the development stage 1 of initial convective development - demonstrates a specific capability of satellite data based detection. It allows for an early detection of pre-thunderstorm convection stages. The nowcasting capabilities of $\mathrm{Cb}$ TRAM are not tested so far. Extrapolation with the disparity vector fields nonetheless illustrate the potential. There is large room for improvement, be it by better taking into account indications of decay or by including some life-cycle model.

The matching and tracking algorithms (TRAM) used here can of course be used for tracking and monitoring of other atmospheric characteristics like precipitation fields from radar data or other types of cloud patterns in satellite images.

\section{Acknowledgements}

We gratefully acknowledge Andreas Dörnbrack who provided the operational analysis data from the ECMWF Mars Archive. The work was supported by the European Commission Projects RISK-AWARE (INTERREG III B CADSES Neighbourhood Programme) and FLYSAVE (6th Framework Programme). Meteosat data are copyrighted by EUMETSAT. The European radar composite is a product of DWD (German Weather Service). We thank two anonymous reviewers for their valuable contributions to the final form of this manuscript.

\section{References}

Arnaud Y, Desbois M, Maizi J (1992) Automatic tracking and characterization of African convective systems on METEOSAT pictures. J Appl Meteorol 31: 443-53

Baker MB, Christian HJ, Latham J (1995) A computational study of the relationship linking lightning frequency and other thundercloud parameters. Q J Roy Meteorol Soc 121: $1525-48$

Bolliger M, Binder P, Rossa A (2003) Tracking cloud patterns by METEOSAT rapid scan imagery in complex terrain. Meteorol Z 12: 73-80

Bugliaro L, Mayer B (2004) Study on quantitative use of the high resolution visible channel onboard the METEOSTAT second generation satellite, Final Report phase II. ITT No. 03/542 EUMETSAT Study EUMETSAT, Am Kavalleriesand 31, 64295 Darmstadt, Germany

Carvalho L, Jones C (2001) A satellite method to identify structural properties of mesoscale convective systems based on the maximum spatial correlation tracking technique (MASCOTTE). J Appl Meteorol 40: 1683-701

Dixon M, Wiener G (1993) TITAN: Thunderstorm identification, tracking, analysis, and nowcasting - a radar-based methodology. J Atmos Ocean Tech 10: 785-97

Ebert EE, McBride JL (2000) Verification of precipitation in weather systems: Determination of systematic errors. J Hydrol 239: 179-202

ECMWF (2005) MARS User Guide European Center for Medium Range Weather Forecast - ECMWF, Reading, 
United Kingdom; Manual available at www.ecmwf.int/ publications/manuals/mars/

Evans JE (1997) Safely reducing delays due to adverse terminal weather. In: Bianco L, Dell'Olmo P, and Odoni AR (eds). Modelling and Simulation in Air Traffic Management. Springer, New York, pp. 185-202

Feidas H, Cartarlis C (2005) Application of an automated cloud-tracking algorithm on satellite imagery for tracking and monitoring small mesoscale convective cloud systems. Int J Remote Sens 26: 1677-98

Fujita T (1969) Present status of cloud velocity computations from ATS-1 and ATS-3 satellites. In: COSPAR Space Research IX, North-Holland, Amsterdam, pp. 557-70

Handwerker J (2002) Cell tracking with TRACE3D - a new algorithm. Atmos Res 61: 15-34

Hering AM, Morel C, Galli G, Senesi S, Ambrosetti P, Boscacci M (2004) Nowcasting thunderstorms in the Alpine Region using a radar based adaptive thresholding scheme. In: Proc. ERAD Conference 2004, Visby, Sweden, pp. 206-11

Horn BKP, Schunck BG (1981) Determining optical flow. Artificial Intelligence 17: 185-203

Lang P (2001) Cell tracking and warning indicators derived from operational radar products. In: Proc. 30th Int. Conference on Radar Meteorology, Munich, Germany, Amer Met Soc, pp. 245-7

Ligda MG (1953) The horizontal motion of small precipitation areas as observed by radar, Tech. Rep. 21. Available from Library, Massachusetts Institute of Technology, 77 Massachusetts Ave., Cambridge, MA, Department of Meteorology, M.I.T.

Lucas BD, and Kanade T (1981) An iterative image registration technique with an application to stereo vision. In: Proc. Imaging Understanding Workshop, pp. 121-30. Available from www-cse.ucsd.edu/classes/sp02/cse252/ lucaskanade81.pdf

Machado LAT, Rossow WB, Guedes RL, Walker AW (1998) Life cycle variations of mesoscale convective systems over the Americas. Mon Wea Rev 126: 1630-54

Mannstein H, Meyer R, Wendling P (1999) Operational detection of contrails from NOAA-AVHRR data. Int $\mathbf{J}$ Remote Sens 20: 1641-60

Mecklenburg S, Joss J, Schmid W (2000) Improving the nowcasting of precipitation in an Alpine region with an enhanced radar echo tracking algorithm. J Hydrol 239: 46-68

Menzel WP (2001) Cloud tracking with satellite imagery: From the pioneering work of Ted Fujita to the present. B Am Meteorol Soc 82: 33-47

Morel C, Senesi S (2002) A climatology of mesoscale convective systems over Europe using satellite infrared imagery. I: Methodology. Q J Roy Meteorol Soc 128: 1953-71

Mueller C, Saxen T, Roberts R, Wilson J, Betancourt T, Dettling S, Oien N, Yee J (2003) NCAR Auto-Nowcast System. Wea Forecast 18: 545-61

Pierce CE, Hardaker PJ, Collier CG, Haggett CM (2000) GANDOLF: a system for generating automated nowcasts of convective precipitation. Meteorol Appl 7: 341-60

Purdom JFW (1976) Some uses of high resolution GOES imagery in the mesoscale forecasting of convection and its behavior. Mon Wea Rev 104: 1474-83

Riosalido R, Carretero O, Elizaga F, Martin F (1998) An experimental tool for mesoscale convective systems tracking. In: Proc. 1st Joint NWC SAF Training Workshop, Madrid

Schmetz J, Holmlund K, Hoffman J, Strauss B, Mason B, Gaertner V, Koch A, Van De Berg L (1993) Operational cloud-motion winds from Meteosat infrared images. J Appl Meteorol 32: 1206-25

Senesi S, Ducrocq V, Thepenier RM, Calas C (1998) MSG and the nowcasting of convective systems: relevance of instability indices and other convection-related diagnostics. In: Proc. 1st Joint NWC SAF Training Workshop, Madrid

Steinacker R, Dorninger M, Wölfelmaier F, Krennert T (2000) Automatic tracking of convective cells and cell complexes from lightning and radar data. Meteorol Atmos Phys 72: 101-10

Takahashi T (1978) Riming electrification as a charge generation mechanism in thunderstorms. J Atmos Sci 35: $1536-48$

Wilson JW, Mueller CK (1993) Nowcasts of thunderstorm initiation and evolution. Wea Forecast 8: 113-31

Wilson JW, Crook NA, Mueller CK, Sun J, Dixon M (1998) Nowcasting thunderstorms: A status report. B Am Meteorol Soc 79: 2079-99

World Meteorological Organisation (1957) Meteorology: a three-dimensional science. WMO Bull 6: 134-8 